\title{
Frontiers in Orthobiologics for the Treatment of Osteochondral Lesions of the Ankle Joint
}

\author{
Ingrid K Stake ${ }^{1}$, Naomasa Fukase ${ }^{2}$, Sudheer Ravuri ${ }^{3}$, C Thomas Haytmanek ${ }^{4}$, Thomas 0 Clanton $^{5}$ and Johnny \\ Huard ${ }^{6 *}$ \\ ${ }^{1}$ Department of Orthopaedic Surgery, Ostfold Hospital Trust, Norway and Steadman Philippon Research Institute, Colorado, USA \\ ${ }^{2}$ Steadman Philippon Research Institute, Colorado and Department of Orthopaedic Surgery, Kobe University Graduate School of Medicine, \\ Japan \\ ${ }^{3}$ Steadman Philippon Research Institute, Colorado, USA
}

${ }^{4}$ The Steadman Clinic, Colorado, USA

${ }^{5}$ The Steadman Clinic and Steadman Philippon Research Institute, Colorado, USA

${ }^{6}$ Steadman Philippon Research Institute, Colorado, USA

*Corresponding author: Johnny Huard, Steadman Philippon Research Institute, 181 W. Meadow Drive, Suite 1000, Vail, Colorado 81657, Colorado, USA

\begin{abstract}
Orthobiologics are receiving increasing interest in an effort to improve cartilage healing after acute and chronic injuries. In osteochondral lesions (OCLs) of the ankle joint, orthobiologics can be used alone or as an adjunct to surgical treatment to optimize the repair tissue. Several treatment options are currently available, including bone and cartilage grafts, cell and/or matrix transplants, and biological adjuncts. However, little consensus exists on how to best use these orthobiologics for optimal repair. The aim of this review was to summarize the latest literature for the treatment of OCLs of the ankle joint, focusing on the most common biological adjuncts; hyaluronic acid (HA), platelet-rich plasma (PRP), and bone marrow aspirate concentrate (BMAC). Additionally, new therapeutic strategies to biologically regulate the cartilage environment to further improve cartilage healing are also discussed.
\end{abstract}

\section{Introduction}

Over the last decade, there has been a growing interest in orthobiologics for osteochondral lesions (OCL) of the ankle joint. Orthobiologics are defined as natural substances in the body that increase the healing process of injury [1]. This includes various bone and cartilage grafts, cell and/or matrix transplants, and biological adjuncts. They can be used as intraarticular injections, administrated to OCLs intraoperatively, or combined with conventional therapies such as debridement or microfracture. Hyaluronic acid (HA), platelet-rich plasma (PRP), and bone marrow aspirate concentrate (BMAC) are common biological adjuncts. The FDA updated its guidance for the use of human cells, tissues, and cellular and tissue-based products (HCT/P) in July 2020[2]. Under these guidelines, PRP is still not considered HCT/P because it is a blood product. Similarly, BMAC is not considered HCT/P as long as it is minimally manipulated, not combined with other substances, and used for homologous purposes. Therefore, neither PRP nor BMAC requires premarket approval by the FDA, which is advantageous for research and therapeutic advancement. OCLs of the ankle joint have been reported in about $50 \%$ of acute ankle sprains and in $73 \%$ of ankle fractures $[3,4]$, thereby representing a frequent problem for the orthopaedic surgeon. If left untreated, the risk of continued symptoms and development of ankle osteoarthritis is considerable, with a reported incidence of osteoarthritis up to $50 \%$ $[5,6]$. However, the treatment of OCLs is difficult due to the poor vascular supply and the large forces that are transferred through the ankle joint. Bone marrow stimulation, or microfracture, is the most commonly used surgical technique for smaller chondral lesions, while osteochondral autograft or allograft transplantation 
has been preferred for larger lesions [3, 5]. Unfortunately, the repair tissue, particularly in microfracture treatment, is primarily composed of fibrocartilage, which is less biomechanically robust than hyaline cartilage, and is more likely to degenerate over time. The aim of this review is to summarize the current literature on orthobiologics for treatment of OCLs of the ankle joint. We have focused on the biological adjuncts, including HA, PRP, and BMAC, with their benefits and limitations based on the latest evidence. Future directions for orthobiologics to promote cartilage repair are also discussed.

\section{Microfracture}

Microfracture surgery is a minimally invasive surgical technique that is easy to perform with low costs [7]. It involves debridement of the unstable cartilage and calcified cartilage layer, and creation of small holes through the subchondral bone to allow for bone marrow with mesenchymal stem cells (MSCs) to fill the chondral defect $[7,8]$. Additionally, a fibrin clot forms within the defect, which initiates an inflammatory state with growth factors and cytokines that stimulate cartilage healing [9]. The MSCs differentiate into chondrocyte-like cells that produce the repair tissue consisting of a combination of hyaline cartilage and fibrocartilage [9-11]. Several studies have reported good clinical outcomes with a low complication rate after microfracture [12-14]. However, the native hyaline cartilage consists of collagen type II, contrary to fibrocartilage, which consists of collagen type I. It has been shown that fibrocartilage has inferior wear properties compared to hyaline cartilage [9]. Declining long-term results with incomplete cartilage healing, progression of osteoarthritis, and poor clinical outcomes have been reported, suggesting that the cartilage may deteriorate over time [15-19].

\section{Bone and Cartilage Grafts}

Osteochondral autograft transplantation (OAT) involves harvest of cartilage graft, usually from the ipsilateral knee joint, with implantation into the cartilage defect [20]. The graft can be harvested as multiple small (mosaicplasty) or one large graft [21]. This procedure thereby replaces the cartilage defect with viable hyaline cartilage. Nevertheless, there is a risk of insufficient healing and symptoms at the donor site as well as cartilage incongruity and poor healing at the graft site [22,23]. Additionally, a medial malleolar or fibular osteotomy is often required to get access to the cartilage defect [20], and poor healing of the osteotomy may be of concern Saxena, et al. [24]. reported comparable functional scores between OAT and microfracture, but longer return to sports with OAT [14]. Although other authors have reported a high rate of good results [20-25], prolonged rehabilitation and risk of complications limit the use of this procedure. Osteochondral allograft transplantation with a graft from a donor avoids the potential complications from the donor site and allows larger grafts to be transplanted; however, limitations include donor accessibility, timing issues, risk of disease transmission, high costs, post-operative cyst formation, not to mention a technically demanding procedure $[3,26]$.

Particulated juvenile cartilage allograft (PJCA) involves transplantation of cartilage from juvenile donors that contains chondrocytes with a higher potential to produce hyaline-like cartilage [27]. Extracellular matrix cartilage allograft (EMCA) consists of a cartilage matrix with various growth factors that can facilitate the differentiation of MSCs to chondrocytes [27]. EMCA has been used as an adjunct to microfracture. Although favorable outcomes have been reported for PJCA and EMCA in both in vitro and clinical studies, the studies are small with wide heterogeneity and several confounding factors [28].

\section{Cell and/or Matrix Transplants}

Cell transplantation aims to replace the chondral defect with hyaline-like cartilage, thereby providing repair tissue with better properties compared to microfracture surgery. Various surgical techniques are described [29]. Autologous chondrocyte implantation (ACI) involves transplantation of cultured chondrocytes $[30,31]$. This is a technically demanding two-stage procedure, where the first stage consists of harvest of chondrocytes from the knee, the ankle, or a loose fragment. After expansion in culture, the chondrocytes are implanted in the lesion and covered with a periosteal flap or type I/III collagen matrix. Matrixassociated chondrocyte implantation (MACI) is also a two-stage procedure $[32,33]$. However, in this procedure, the chondrocytes are cultured on a type I/III collagen matrix, thereby reducing the risk of chondrocyte dedifferentiation, avoiding the need for a covering scaffold, and promoting an even chondrocyte distribution. Autologous matrix-induced chondrogenesis (AMIC) is also used as an adjunct to microfracture [34]. After the microfracture procedure, a type I/III collagen matrix is used to cover the cartilage defect. The matrix is thought to prevent the fibrin clot and MSCs from escaping and to stimulate chondrogenesis [35]. Since no cells are transplanted, this procedure relies on the differentiation of the MSCs for production of repair tissue. Matrix-associated stem cell transplantation (MAST) is a modification of the AMIC procedure. Autologous bone marrow aspirate is harvested from the iliac crest, concentrated, and impacted into the cartilage lesion together with a type I/III collagen matrix or extracellular matrix (ECM) scaffold [36]. This procedure thereby requires only one stage and results in a higher number of MSCs available for cartilage repair.

Clinical studies have reported promising outcomes after autologous cell transplantation; however, most of the literature consists of smaller case series with wide heterogeneity [35-39]. There is frequently a need for a medial malleolar osteotomy, and both ACI and MACI consist of two procedures leading to increased morbidity and costs. Additionally, there are regulations 
on sorting and culturing of cells by the FDA in the United States. These limitations have prevented the widespread usage of cell transplantation.

\section{Biological Adjuncts \\ Hyaluronic Acid}

Hyaluronic acid (HA) is a carbohydrate component found in the synovial fluid that provides viscoelastic properties [40]. In laboratory studies, HA has demonstrated a positive effect on chondrocyte proliferation [41]. Given as intraarticular injections for OCLs of the ankle joint, HA serves as a visco-supplementation and has been reported to reduce pain and improve function [42]. In a randomized controlled trial by Doral et al. 41 patients were treated with three HA injections after microfracture surgery and 16 patients were treated with microfracture surgery only [40]. After 2 years, they found a significantly better improvement in function measured with the Freiburg score and the American Orthopaedic Foot and Ankle Society (AOFAS) score with postoperative HA. No complications were reported. Similarly, in a randomized controlled trial, Shang et al. compared 18 patients treated with microfracture and three HA injections with 17 patients treated with microfracture alone [43]. In addition to better AOFAS and visual analogue scale (VAS) scores in the HA group, they reported magnetic resonance imaging (MRI) findings suggesting better remodeling and maturation of the repair tissue at a mean 10 months postoperatively. Although lack of long-term results, there is evidence that HA injections as an adjunct to microfracture surgery may have superior results compared to microfracture alone.

\section{Platelet-Rich Plasma}

Platelet-rich plasma (PRP) consists of a concentrate of platelets and is derived from peripheral venous blood that is processed and separated into blood fractions. Platelets contain a high volume of cytokines and growth factors, including transforming growth factor beta (TGF-ß), vascular endothelial growth factor (VEGF), plateletderived growth factor (PDGF), insulin-like growth factor (IGF), and fibroblast growth factor (FGF) [44]. These are released when the platelets are activated by exogenous or endogenous triggers. The cytokines and growth factors play an essential role in the homeostatic, coagulatory, and inflammatory processes.

A systematic review by Smyth et al. included in vitro and in vivo studies that examined the effect of PRP on cartilage [45]. Most of the included studies found evidence for increased cell proliferation, differentiation, and viability, and they also found higher levels of proteoglycan and type II collagen. This may be beneficial following the microfracture procedure, where PRP may improve the amount and quality of repair tissue. Milano et al. studied chronic OCLs in the weightbearing area of the knee in 15 sheep [46]. The lesions were treated with microfracture alone, microfracture with PRP and fibrin glue, or microfracture and one PRP injection. Six months postoperatively, the PRP and fibrin glue group showed better covering by repair tissue, higher stiffness, and better histological findings than the two other groups. Also, the PRP injection group demonstrated better histological findings than the microfracture group. Smyth et al. assessed PRP as an adjunct to OAT [47]. In each of 12 rabbits, one knee was treated with OAT and saline injection and the contralateral knee with OAT and PRP injection. No difference between the treatments was found macroscopically, however, the histological score and graft integration were better in the PRP group at 12 weeks postoperatively. Based on the pre-clinical studies, PRP as an adjunct to surgical treatment for OCLs may result in superior repair tissue compared to microfracture surgery.

Some clinical studies have reported on PRP treatment alone or as an adjunct to microfracture surgery in the ankle joint. A randomized controlled trial by Mei-Dan et al. compared three injections of PRP or HA in 30 ankles [48]. They reported a significant improvement in AOFAS score and VAS scores for pain, stiffness, and function in both groups at 28 weeks, although PRP demonstrated significantly better results compared to HA. Guney, et al. compared microfracture alone $(n=16)$ to microfracture and one PRP injection $(n=19)$ [49]. At a minimum of 12 months postoperatively, the AOFAS, VAS, and Foot and Ankle Ability Measure (FAAM) scores improved significantly in both groups. Additionally, the improvement was significantly better with microfracture and PRP compared to microfracture alone. Similar findings were reported by Görmeli, et al. [50]. They compared one injection of either PRP, HA, or placebo after microfracture surgery in a blinded randomized controlled trial including 40 patients. At mean 15.3 months, both AOFAS and VAS scores improved significantly in all groups, with a significantly better improvement in the PRP group compared to the HA group, and in the HA group compared to the placebo group. In a study by Guney et al., microfracture alone ( $\mathrm{n}=$ 19), microfracture and one PRP injection ( $\mathrm{n}=22)$, and mosaicplasty ( $n=13$ ) were compared [51]. Mosaicplasty was chosen for larger lesions in patients with high physical demands. At a median followup of 42 months, the mosaicplasty group had a significantly larger reduction in VAS score for pain compared to the two other groups, but these patients also had higher VAS score preoperatively. AOFAS and FAAM scores did not differ between the groups. Interestingly, five patients in the microfracture group had reoperation due to pain. The authors suggested that better quality of the repair tissue might favor mosaicplasty when comparing clinical outcomes.

PRP has also been used as an adjunct to other surgical treatments for OCLs. Giannini et al. treated 48 patients with a combination of PRP gel and autologous bone marrow-derived cells on a porcine collagen or HA membrane placed in the cartilage defect [52]. At 12 months follow-up, five second-look arthroscopies demonstrated intact repair cartilage and histologic findings with various degrees of repair tissue. At 24 months follow-up, a high AOFAS score with healing on MRI was reported. In a follow-up study by the same 
authors, comparable results were found between this technique, open ACI, and arthroscopic ACI [53]. Gu et al. reported on PRP as a scaffold placed over autologous bone graft [54]. At mean 18 months (range, 12-24) follow-up, they found a significant increase in AOFAS, VAS, and SF-36 scores, and improvement of dorsi- and plantarflexion.

PRP treatment is easy to perform, involves a low risk of complications, and has low costs. Today, several commercial systems are available for processing PRP to obtain the desired composition of blood fractions (e.g., leukocyte-rich PRP and leukocyte-poor PRP). However, these systems prepare PRP with various concentrations of products (i.e., platelets, leukocytes, anticoagulants, activators) [55]. Additionally, patient characteristics, including age and body mass index (BMI), may result in different PRP compositions. There is also inconsistency regarding dosages, timing, and frequency of treatment [56]. The diversity of PRPs, as well as the wide heterogeneity of methodology in existing studies, make comparisons between studies and interpretation of results difficult.

\section{Bone Marrow Aspirate Concentrate}

Bone marrow aspirate concentrate (BMAC) is designed to enrich the tissue with white blood cells, platelets, and MSCs, and is created by centrifugation of collected autologous bone marrow [57]. Bone marrow can be harvested from multiple anatomical sites such as the anterior iliac crest, posterior iliac crest, tibia, and calcaneus [58]. Among several orthobiologics routinely used as adjuncts in clinical practice, BMAC initially gained popularity as an intraoperative stem cell therapy due to the characteristics of being harvested from bone marrow; the primary source of MSCs [59]. Since MSCs have the ability to differentiate into different cell types depending on the environment, they can be expected to differentiate into chondrocytes when implanted into OCLs [60]. However, there is a controversial question whether BMAC administration can be considered a stem cell therapy. This is due to recent evidence of low concentrations of MSCs in BMACs $(0.001 \%$ to $0.01 \%$ ) [61,62]. Furthermore, in a recently published systematic review investigating the benefit of BMAC in focal cartilage lesions of the knee joint, Cavinatto et al., [63]. found that there was no evidence of presence of true MSCs in BMAC. Also, recent studies have shown that MSCs do not differentiate directly into cartilage, but rather release microvesicles that act to regulate the local environment, thus leading to recruitment of more stem cells [64, 65]. Interestingly, these cells may possess a paracrine effect that recruits additional host cells to the delivery site, enhancing the production of additional growth factors to promote tissue healing [63].

BMAC is also known to contain multiple growth factors, including TGF- $\beta$, VEGF, PDGF, bone morphogenetic proteins (BMPs), and interleukin-8 (IL-8) [66]. Despite similar platelet concentration in BMAC and PRP, BMAC contains a significant amount of leukocytes and the pro-inflammatory cytokines IL-8 and IL-1 $\beta$ [64]. Furthermore, the recent finding that BMAC contains significantly higher concentrations of the interleukin-1 receptor antagonist (IL-1ra), an anti-inflammatory protein, strongly supports the antiinflammatory effect of BMAC [59, 64]. Given that inflammatory suppression has always been the key to cartilage repair [67], BMAC may have an advantage over PRP for OCL repair.

Over the past decade, several clinical studies have been conducted to evaluate the efficacy of BMAC for the treatment of OCLs of the ankle joint, mainly administered in situ and combined with various scaffolds, grafting techniques, or microfracture procedures [20, 52, 68-73]. In their one-step repair using BMAC in talar OCLs, Giannini et al. showed good clinical outcomes at 4-year follow-up, despite a decrease in the AOFAS scores between 24 and 48 months after surgery [69]. Their results suggest that BMAC is a useful biologic for the regeneration of damaged cartilage in the talus. However, further long-term follow-up is necessary to determine the long-term durability of the regenerated cartilage. Hannon et al. retrospectively compared the outcomes of patients who received microfracture and BMAC $(n=22)$ or microfracture alone $(n=12)$ for treatment of OCL of the talus [68]. The authors found that the microfracture and BMAC procedures produced similar favorable functional outcomes to microfracture alone, but with improved integration of borderline repair tissue as well as fewer tears and fibrillation on MRI.

Clinical studies have also been conducted with BMAC in combination with autograft or allograft transplantation to achieve higher quality cartilage regeneration. Kennedy et al. reported functional outcomes after autologous osteochondral transplantation using BMAC in 72 patients [20]. At an average of 28 months postoperatively, the authors described favorable functional outcome improvements and concluded that autologous osteochondral grafting is a reproducible primary therapeutic strategy for large OCLs of the talus. Recently, De Sandis, et al. assessed clinical and radiographic outcomes in 46 patients with critical size OCLs ( $\geq 6 \mathrm{~mm}$ widest diameter) who underwent Juvenile Allogenic Chondrocyte Implantation (JACI) with BMAC [73]. The results showed that the combination of JACI and BMAC improved functional outcome scores, however, postoperative MRI showed low surface continuity (9.1\%), homogenous structure (13.6\%), and poor cartilage integration (40.9\%) which still presented a fibrocartilage configuration. A retrospective study was recently published from the same authors, comparing three groups of patients who underwent JACI with BMAC (JACI-BMAC, $n=20$ ), microfracture alone (MF, $n=13)$, or microfracture with BMAC (BMAC-MF, $n=17$ ) [72]. They found that functional outcomes 
improved at an average of 30.9 months after surgery in all three groups. Although most patients improved, functional outcomes and quality of repair tissue were still abnormal. Furthermore, the JACI-BMAC group showed fibrocartilage regeneration on MRI after implantation and did not show significant functional superiority compared to the MF group. Based on these results, there is no evidence that this relatively new grafting technique combined with BMAC is advantageous compared to other conventional cartilage repair techniques such as microfracture.

There is growing evidence on the use of BMAC to treat OCLs of the ankle joint. However, few publications demonstrate the efficacy of BMAC alone, and it has yet to be known whether BMAC can provide temporary pain relief or long-term patient benefits. High-quality studies assessing outcome selection, processing, and administration are essential to determine the clinical benefits of BMAC.

\section{Future Directions for OCL Treatment of the Ankle Joint}

Healing of OCLs with hyaline cartilage that is equivalent to native cartilage is crucial to withstand the high mechanical stress over the long term and avoid the development of osteoarthritis of the ankle joint. Numerous studies have examined the possibility of enhancing cartilage repair with scaffolds, growth factors, or stem cells, either alone or as an adjunct to conventional surgical techniques. Unfortunately, none of the available treatment options today results in high-quality hyaline cartilage. Optimizing the environment for cartilage repair or the composition of the biological adjuncts may allow for further increased quality of the repair tissue.

TGF-ß1 plays a vital role in cartilage homeostasis [74]. However, it is also known that TGF- $\beta 1-p S m a d 2 / 3$ signaling is associated with tissue fibrosis [74]. Some studies have demonstrated that reducing the level of TGF- $ß 1$ through blocking of angiotensin II receptors can reduce tissue fibrosis and increases tissue regeneration after skeletal muscle injury [75-77]. Recently, Utsunomiya et al. showed in a rabbit knee model that losartan, a well-known angiotensin II receptor blocker, reduced the level of TGF- $\beta 1$ and resulted in improved cartilage repair with increased hyaline cartilage after microfracture procedure [78].

VEGF is a cytokine that stimulates angiogenesis and is highly important during inflammation and bone healing [79]. It has been suggested that inhibition of VEGF signaling may inhibit the angiogenesis, thereby reduce the progression of osteoarthritis and improve the healing of OCLs [80, 81]. In a rabbit knee osteochondral defect model, Nagai, et al. reported that intravenous bevacizumab, an anti-VEGF drug, significantly improved the healing of osteochondral defects compared to untreated controls [81].

PRP and BMAC contain growth factors and cytokines that act negatively on the healing process, including pro-inflammatory interleukins, microparticles, and reactive oxygen species (ROS) [82,
83]. These components may potentially be modified to improve healing. Additionally, senescent cells are present in both PRP and BMAC, and release pro-inflammatory cytokines, proteases, and other senescent-associated secretory phenotypes (SASPs), which may reduce the quality of tissue repair. $\mathrm{Xu}$, et al. reported that transplanting senescent cells into the knee joint caused severe articular cartilage damage suggestive of osteoarthritis, indicating that senescent cells can induce an osteoarthritis-like state [84]. Administration of senolytic agents to remove senescent cells and reduce SASP may enhance the effect of these orthobiologics and thus provide a safe and novel therapeutic strategy.

\section{Conclusion}

After surgical treatment of OCLs of the ankle joint, regeneration with hyaline cartilage is essential to withstand repetitive stress from weightbearing. Although orthobiologics are increasingly used to improve cartilage healing, there is insufficient evidence for their indications, preparation, dosage, and timing. Additionally, comparative studies with long-term follow-up to determine the quality and durability of the repaired cartilage are needed before definitive treatment recommendations can be made.

\section{References}

1. American Academy of Orthopaedic Surgeons. Helping Fractures Heal (Orthobiologics). Januar 2010 August 2020]

2. (2020) FDA Guidance for Industry and Food and Drug Administration Staff. Regulatory Considerations for Human Cells, Tissues, and Cellular and Tissue-Based Products: Minimal Manipulation and Homologous Use.

3. Murawski CD, Kennedy JG (2013) Operative treatment of osteochondral lesions of the talus. J Bone Joint Surg Am 95(11): 1045-1054.

4. Leontaritis N, Hinojosa L, Panchbhavi VK (2009) Arthroscopically detected intra-articular lesions associated with acute ankle fractures. J Bone Joint Surg Am 91(2): 333-339.

5. Zengerink, M, Peter A A Struijs, Johannes L Tol, Cornelis Niek van Dijk (2010) Treatment of osteochondral lesions of the talus: a systematic review. Knee Surg Sports Traumatol Arthrosc 18(2): 238-246.

6. Canale ST, RH Belding (1980) Osteochondral lesions of the talus. J Bone Joint Surg Am 62(1): 97-102.

7. Steadman JR, Rodkey WG, Rodrigo JJ (2001) Microfracture: surgical technique and rehabilitation to treat chondral defects. Clin Orthop Relat Res (391 Suppl): S362-369.

8. Frisbie DD, Sophie Morisset, Charles P Ho, William G Rodkey, J Richard Steadman, et al. (2006) Effects of calcified cartilage on healing of chondral defects treated with microfracture in horses. Am J Sports Med 34(11): 1824-1831.

9. Mankin HJ (1982) the response of articular cartilage to mechanical injury. J Bone Joint Surg Am 64(3): 460-466.

10. Frisbie DD, Julia T Oxford, Louise Southwood, Gayle W Trotter, William G Rodkey, et al. [2003] Early events in cartilage repair after subchondral bone microfracture. Clin Orthop Relat Res 407: 215-227.

11. Frisbie DD, Trotter GW, Powers BE, Rodkey WG, Steadman JR, et al. (1999) Arthroscopic subchondral bone plate microfracture technique augments healing of large chondral defects in the radial carpal bone and medial femoral condyle of horses. Vet Surg 28(4): 242-255. 
12. Donnenwerth, MP, Roukis TS (2012) Outcome of arthroscopic debridement and microfracture as the primary treatment for osteochondral lesions of the talar dome. Arthroscopy 28(12): 19021907.

13. Clanton TO, Johnson NS, Matheny LM (2014) Outcomes Following Microfracture in Grade 3 and 4 Articular Cartilage Lesions of the Ankle. Foot Ankle Int 35(8): 764-770.

14. Saxena A, Eakin C (2007) Articular talar injuries in athletes: results of microfracture and autogenous bone graft. Am J Sports Med 35(10): 1680-1687.

15. Keun-Bae Lee, Long-Bin Bai, Taek-Rim Yoon, Sung-Taek Jung, JongKeun Seon, et al. (2009) Second-look arthroscopic findings and clinical outcomes after microfracture for osteochondral lesions of the talus. Am J Sports Med 37: 63S-70S.

16. Ferkel R.D, Robert M Zanotti, Gregory A Komenda, Nicholas A Sgaglione, Margaret S Cheng, et al. (2008) Arthroscopic treatment of chronic osteochondral lesions of the talus: long-term results. Am J Sports Med 36(9): 1750-1762.

17. Becher C, David Zühlke, Christian Plaas, Marc Ewig, Tilman Calliess, et al. (2015) T2-mapping at $3 \mathrm{~T}$ after microfracture in the treatment of osteochondral defects of the talus at an average follow-up of 8 years. Knee Surg Sports Traumatol Arthrosc 23(8): 2406-2412.

18. Bae DK, Sang Jun Song, Kyoung Ho Yoon, Dong Beom Heo, Tae Jin Kim, et al. (2013) Survival analysis of microfracture in the osteoarthritic kneeminimum 10-year follow-up. Arthroscopy, 29(2): 244-250.

19. Seow D, Youichi Y, Eoghan TH, Yoshiharu S, Hutchinson ID, et al. (2019) The Subchondral Bone Is Affected by Bone Marrow Stimulation: A Systematic Review of Preclinical Animal Studies. Cartilage 10(1): 70-81.

20. Kennedy JG, Murawski CD (2011) The Treatment of Osteochondral Lesions of the Talus with Autologous Osteochondral Transplantation and Bone Marrow Aspirate Concentrate: Surgical Technique. Cartilage 2(4): 327-336.

21. Hangody, L, Gábor Vásárhelyi, László Rudolf Hangody, Zita Sükösd, György Tibay, et al. (2008) Autologous osteochondral grafting-technique and long-term results. Injury 39(1): S32-39.

22. Reddy S, David I Pedowitz, Selene G Parekh, Brian J Sennett, Enyi Okereke, et al. (2007) The morbidity associated with osteochondral harvest from asymptomatic knees for the treatment of osteochondral lesions of the talus. Am J Sports Med 35(1): 80-85.

23. LaPrade RF, Botker JC (2004) Donor-site morbidity after osteochondral autograft transfer procedures. Arthroscopy 20(7): e69-e73.

24. Lamb J, Christopher D Murawski, Timothy W Deyer, John G Kennedy (2013) Chevron-type medial malleolar osteotomy: a functional, radiographic and quantitative T2-mapping MRI analysis. Knee Surg Sports Traumatol Arthrosc 21(6): 1283-1288.

25. Gobbi A, Ramces AF, James HL, Francesco A, Gianluigi Canata, et al (2006) Osteochondral lesions of the talus: randomized controlled trial comparing chondroplasty, microfracture, and osteochondral autograft transplantation. Arthroscopy 22(10): 1085-1092.

26. Ackermann J, Gergo Merkely, Nehal Shah, Andreas H Gomoll (2019) Decreased Graft Thickness Is Associated With Subchondral Cyst Formation After Osteochondral Allograft Transplantation in the Knee. Am J Sports Med 47(9): 2123-2129.

27. West TA, Williams ML (2019) Orthobiologics. Clin Podiatr Med Surg 36(4): 609-626.

28. Seow D, Youichi Yasui, Eoghan T Hurley, Andrew W Ross, Christopher D M, et al. (2018) Extracellular Matrix Cartilage Allograft and Particulate Cartilage Allograft for Osteochondral Lesions of the Knee and Ankle Joints: A Systematic Review. Am J Sports Med, 2018. 46(7): 1758-1766.
29. McGoldrick NP, Murphy EP, Kearns SR (2018) Osteochondral lesions of the ankle: The current evidence supporting scaffold-based techniques and biological adjuncts. Foot Ankle Surg, 2018. 24(2): 86-91.

30. Brittberg M, A Lindahl, A Nilsson, C Ohlsson, O Isaksson, et al. (1994) Treatment of deep cartilage defects in the knee with autologous chondrocyte transplantation. N Engl J Med 331(14): 889-895.

31. Petersen L, Brittberg M, Lindahl A (2003) Autologous chondrocyte transplantation of the ankle. Foot and Ankle Clinics 8(2): 291-303.

32. Cherubino P, Grassi FA, Bulgheroni P, Ronga M, et al. (2003) Autologous chondrocyte implantation using a bilayer collagen membrane: a preliminary report. J Orthop Surg (Hong Kong) 11(1): 10-15.

33. Clanton TO, Johnson NS, Matheny LM (2014) Use of Cartilage Extracellular Matrix and Bone Marrow Aspirate Concentrate in Treatment of Osteochondral Lesions of the Talus. Techniques in Foot \& Ankle Surgery 13(4): 212-220.

34. Benthien JP, Behrens P (2010) Autologous Matrix-Induced Chondrogenesis (AMIC): Combining Microfracturing and a Collagen I/III Matrix for Articular Cartilage Resurfacing. Cartilage 1(1): 65-68.

35. Gao L, Patrick Orth, Magali Cucchiarini, Henning Madry (2019) Autologous Matrix-Induced Chondrogenesis: A Systematic Review of the Clinical Evidence. Am J Sports Med 47(1): 222-231.

36. Richter M, Zech S (2013) Matrix-associated stem cell transplantation (MAST) in chondral defects of foot and ankle is effective. Foot Ankle Surg 19(2): 84-90.

37. Erickson B, Yale Fillingham, Michel Hellam, Selene G, Christopher, et al. (2018) Surgical management of large talar osteochondral defects using autologous chondrocyte implantation. Foot Ankle Surg 24(2): 131-136.

38. Murphy EP, Christopher Fenelon, Ciara Egan, Stephen R (2019) Matrixassociated stem cell transplantation is successful in treating talar osteochondral lesions. Knee Surg Sports Traumatol Arthrosc 27(9): 2737-2743.

39. Richter M, Zech S (2019) Matrix-associated stem cell transplantation (MAST) in chondral lesions at the ankle as part of a complex surgical approach- 5-year-follow-up in 100 patients. Foot Ankle Surg 25(3): 264271.

40. Doral MN, O Bilge, G Batmaz, G Donmez, E Turhan, et al. (2012) Treatment of osteochondral lesions of the talus with microfracture technique and postoperative hyaluronan injection. Knee Surg Sports Traumatol Arthrosc 20(7): 1398-1403.

41. Kawasaki K, Ochi M, Uchio Y, Adachi N, Matsusaki M, et al. (1999) Hyaluronic acid enhances proliferation and chondroitin sulfate synthesis in cultured chondrocytes embedded in collagen gels. J Cell Physiol 179(2): 142-148.

42. Mei-Dan 0, Guy Maoz, Michael Swartzon, Erol Onel, Beni Kish, et al. (2008) Treatment of osteochondritis dissecans of the ankle with hyaluronic acid injections: a prospective study. Foot Ankle Int 29(12): 1171-1178.

43. Shang XL, Hong-Yue Tao, Shi-Yi Chen, Yua-Xia Li, Ying-Hui Hua, et al.(2016) Clinical and MRI outcomes of HA injection following arthroscopic microfracture for osteochondral lesions of the talus. Knee Surg Sports Traumatol Arthrosc 24(4): 1243-1249.

44. Fortier LA, Joseph U Barker, Eric J Strauss, Taralyn M McCarrel, Brian J Cole, et al. (2011) The role of growth factors in cartilage repair. Clin Orthop Relat Res 469(10): 2706-2715.

45. Smyth NA, Christopher D Murawski, Lisa A Fortier, Brian J Cole, John G Kennedy, et al. (2013) Platelet-rich plasma in the pathologic processes of cartilage: review of basic science evidence. Arthroscopy 29(8): 13991409. 
46. Milano G, E Sanna Passino, L Deriu, G Careddu, L Manunta, A Manunta, et al. (2010) The effect of platelet rich plasma combined with microfractures on the treatment of chondral defects: an experimental study in a sheep model. Osteoarthritis Cartilage 18(7): 971-980.

47. Smyth NA, Amgad M Haleem, Christopher D Murawski, Huong T Do, Jonathan T Deland, et al. (2013) The effect of platelet-rich plasma on autologous osteochondral transplantation: an in vivo rabbit model. J Bone Joint Surg Am 95(24): 2185-2193.

48. Mei-Dan O, Michael R Carmont, Lior Laver, Gideon Mann, Nicola Maffull, Meir Nyska, et al. (2012) Platelet-rich plasma or hyaluronate in the management of osteochondral lesions of the talus. Am J Sports Med 40(3): 534-541.

49. Guney A, Mustafa Akar, Ibrahim Karaman, Mithat Oner, Betul Guney et al. (2015) Clinical outcomes of platelet rich plasma (PRP) as an adjunct to microfracture surgery in osteochondral lesions of the talus. Knee Surg Sports Traumatol Arthrosc 23(8): 2384-2389.

50. Gormeli, G, Mustafa Karakaplan, Cemile Ayşe Görmeli, Baran Sarıkaya, Nurzat Elmalı et al. (2015) Clinical Effects of PlateletRich Plasma and Hyaluronic Acid as an Additional Therapy for Talar Osteochondral Lesions Treated with Microfracture Surgery: A Prospective Randomized Clinical Trial. Foot Ankle Int 36(8): 891-900.

51. Guney A, Emre Yurdakul, Ibrahim Karaman, Okkes Bilal, Ibrahim Halil Kafadar, et al. (2016) Medium-term outcomes of mosaicplasty versus arthroscopic microfracture with or without platelet-rich plasma in the treatment of osteochondral lesions of the talus. Knee Surg Sports Traumatol Arthrosc 24(4): 1293-1298

52. Giannini S, Roberto Buda, Francesca Vannini, Marco Cavallo, Brunella Grigolo, et al. (2009) One-step bone marrow-derived cell transplantation in talar osteochondral lesions. Clin Orthop Relat Res 467(12): 33073320 .

53. Giannini S, Roberto Buda, Marco Cavallo, Alberto Ruffilli, Annarita Cenacchi, et al. (2010) Cartilage repair evolution in post-traumatic osteochondral lesions of the talus: from open field autologous chondrocyte to bone-marrow-derived cells transplantation. Injury 41(11): 1196-1203.

54. Gu W, Tanzhu Li, Zhongmin Shi, Guohua Mei, Jianfeng Xue, et al. (2017) Management of Hepple Stage V Osteochondral Lesion of the Talus with a Platelet-Rich Plasma Scaffold. Biomed Res Int 2017: 6525373.

55. Vannini, F, Di Matteo B, Filardo G, Kon E, Marcacci M, et al. (2014) Platelet-rich plasma for foot and ankle pathologies: a systematic review. Foot Ankle Surg 20(1): 2-9.

56. Boffa A, Davide Previtali, Sante Alessandro Altamura, Stefano Zaffagnini, Christian Candrian, et al. (2020) Platelet-Rich Plasma Augmentation to Microfracture Provides a Limited Benefit for the Treatment of Cartilage Lesions: A Meta-analysis. Orthop J Sports Med 8(4): p. 2325967120910504.

57. Holton J, Mohamed Imam, Jonathan Ward, Martyn Snow, et al. (2016) The Basic Science of Bone Marrow Aspirate Concentrate in Chondral Injuries. Orthop Rev (Pavia) 8(3): 6659.

58. Hyer CF, Gregory C Berlet, Bradly W Bussewitz, Thomas Hankins, Heidi L Ziegler, et al. (2013) Quantitative assessment of the yield of osteoblastic connective tissue progenitors in bone marrow aspirate from the iliac crest, tibia, and calcaneus. J Bone Joint Surg Am, 2013. 95(14): 13121316.

59. Fortier LA, Eric J Strauss, David O Shepard, Liliya Becktell, John G Kennedy, et al. (2019) Biological Effects of Bone Marrow Concentrate in Knee Pathologies. J Knee Surg 32(1): 2-8.

60. Cottom JM, Plemmons BS (2018) Bone Marrow Aspirate Concentrate and Its Uses in the Foot and Ankle. Clin Podiatr Med Surg 35(1): 19-26.

61. Madry H, Liang Gao, Hermann Eichler, Patrick Orth, Magali Cucchiarini et al. (2017) Bone Marrow Aspirate Concentrate-Enhanced Marrow Stimulation of Chondral Defects. Stem Cells Int 2017: 1609685.
62. Cotter EJ, Kevin C Wang, Adam B Yanke, Susan Chubinskaya, et al. (2018) Bone Marrow Aspirate Concentrate for Cartilage Defects of the Knee: From Bench to Bedside Evidence. Cartilage 9(2): 161-170.

63. Cavinatto L, Betina B Hinckel, Ryan E Tomlinson, Sunny Gupta, Jack Farr, et al. (2019) The Role of Bone Marrow Aspirate Concentrate for the Treatment of Focal Chondral Lesions of the Knee: A Systematic Review and Critical Analysis of Animal and Clinical Studies. Arthroscopy, 35(6): 1860-1877.

64. Cassano JM, John G Kennedy, Keir A Ross, Ethan J Fraser, Margaret B Goodaleet al. (2018) Bone marrow concentrate and platelet-rich plasma differ in cell distribution and interleukin 1 receptor antagonist protein concentration. Knee Surg Sports Traumatol Arthrosc 26(1): 333-342.

65. De Bari C, Roelofs AJ (2018) Stem cell-based therapeutic strategies for cartilage defects and osteoarthritis. Curr Opin Pharmacol 40: 74-80.

66. Harford JS, Dekker TJ, Adams SB (2016) Bone Marrow Aspirate Concentrate for Bone Healing in Foot and Ankle Surgery. Foot Ankle Clin 21(4): 839-845

67. Zhang Y, Pizzute T, Pei M (2014) Anti-inflammatory strategies in cartilage repair. Tissue Eng Part B Rev 20(6): 655-668.

68. Hannon CP, Keir A Ross, Christopher D Murawski, Timothy W Deyer, Niall A Smyth, et al. (2016) Arthroscopic Bone Marrow Stimulation and Concentrated Bone Marrow Aspirate for Osteochondral Lesions of the Talus: A Case-Control Study of Functional and Magnetic Resonance Observation of Cartilage Repair Tissue Outcomes. Arthroscopy 32(2): 339-347.

69. Giannini S, Roberto Buda, Milva Battaglia, Marco Cavallo, Alberto Ruffilli, et al. (2013) One-step repair in talar osteochondral lesions: 4-year clinical results and t2-mapping capability in outcome prediction. Am J Sports Med 41(3): 511-518.

70. Shimozono Y, Youichi Yasui, Eoghan T Hurley, Rebecca A Paugh, Timothy W Deyer, et al. (2019) Concentrated Bone Marrow Aspirate May Decrease Postoperative Cyst Occurrence Rate in Autologous Osteochondral Transplantation for Osteochondral Lesions of the Talus. Arthroscopy 35(1): 99-105.

71. Bieback K, H Kluter (2007) Mesenchymal stromal cells from umbilical cord blood. Curr Stem Cell Res Ther 2(4): 310-323.

72. Karnovsky SC, Bridget DeSandis, Amgad M Haleem, Carolyn M Sofka, Martin O'Malley, et al. (2018) Comparison of Juvenile Allogenous Articular Cartilage and Bone Marrow Aspirate Concentrate Versus Microfracture With and Without Bone Marrow Aspirate Concentrate in Arthroscopic Treatment of Talar Osteochondral Lesions. Foot Ankle Int 39(4): 393-405.

73. DeSandis BA, Amgad M Haleem, Carolyn M Sofka, Martin J O’Malley, Mark C Drakos, et al. (2018) Arthroscopic Treatment of Osteochondral Lesions of the Talus Using Juvenile Articular Cartilage Allograft and Autologous Bone Marrow Aspirate Concentration. J Foot Ankle Surg 57(2): 273-280.

74. Blaney Davidson EN, van der Kraan PM, van den Berg WB (2007) TGFbeta and osteoarthritis. Osteoarthritis Cartilage 15(6): 597-604.

75. Bedair HS, Tharun Karthikeyan, Andres Quintero, Yong Li, Johnny Huard, et al. (2008) Angiotensin II receptor blockade administered after injury improves muscle regeneration and decreases fibrosis in normal skeletal muscle. Am J Sports Med 36(8): 1548-1554.

76. Kobayashi M, Shusuke Ota, Satoshi Terada, Yohei Kawakami, Takanobu Otsuka, et al. (2016) The Combined Use of Losartan and Muscle-Derived Stem Cells Significantly Improves the Functional Recovery of Muscle in a Young Mouse Model of Contusion Injuries. Am J Sports Med 44(12): 3252-3261.

77. Terada S, Shusuke Ota, Makoto Kobayashi, Tetsuo Kobayashi, Yutaka Mifune, et al. (2013) Use of an antifibrotic agent improves the effect of platelet-rich plasma on muscle healing after injury. J Bone Joint Surg Am 95(11): 980-988. 
78. Utsunomiya H, Xueqin Gao, Zhenhan Deng, Haizi Cheng, Gilberto Nakama, et al. (2020) Biologically Regulated Marrow Stimulation by Blocking TGF-beta1 With Losartan Oral Administration Results in Hyaline-like Cartilage Repair: A Rabbit Osteochondral Defect Model. Am J Sports Med 48(4): 974-984.

79. Hu K, Olsen BR (2016) The roles of vascular endothelial growth factor in bone repair and regeneration. Bone 91: 30-38.

80. Nagai T, Masato Sato, Miyuki Kobayashi, Munetaka Yokoyama, Yoshiki Tani, et al. (2014) Bevacizumab, an anti-vascular endothelial growth factor antibody, inhibits osteoarthritis. Arthritis Res Ther 16(5): p. 427.

81. Nagai T, Masato Sato, Toshiharu Kutsuna, Mami Kokubo, Goro Ebihara, et al. (2010) Intravenous administration of anti-vascular endothelial growth factor humanized monoclonal antibody bevacizumab improves articular cartilage repair. Arthritis Res Ther 12(5): R178.
82. Hudgens JL, Kristoffer B Sugg, Jeremy A Grekin, Jonathan P Gumucio, Asheesh Bedi, et al. (2016) Platelet-Rich Plasma Activates Proinflammatory Signaling Pathways and Induces Oxidative Stress in Tendon Fibroblasts. Am J Sports Med 44(8): 1931-1940.

83. Ziegler CG, Rachel Van Sloun, Sabrina G, Kaitlyn EW, Nicolos N, et al., Characterization of Growth Factors, Cytokines, and Chemokines in Bone Marrow Concentrate and Platelet-Rich Plasma: A Prospective Analysis. Am J Sports Med 47(9): 2174-2187.

84. Farr JN, Ming Xu, Meghan M Weivoda, David GM, Daniel GF, et al. (2017) Targeting cellular senescence prevents age-related bone loss in mice. Nat Med 23(9): 1072-1079.

\section{(C) This work is licensed under Creative}

To Submit Your Article Click Here: Submit Article

DOI: $10.32474 /$ OSMOAJ.2020.04.000188

$\begin{gathered}\text { Orthopedics and Sports Medicine } \\ \text { Open Access Journal }\end{gathered}$
Assets of Publishing with us
- Global archiving of articles
- Immediate, unrestricted online access
- Rigorous Peer Review Process
- Authors Retain Copyrights
Orthopedics And Sports Medicine:
Open Access Junrnal

